\title{
Qing Hua Chang Yin exerts therapeutic effects against ulcerative colitis through the inhibition of the TLR4/NF-kB pathway
}

\author{
XIAO KE ${ }^{1}$, FAN ZHOU ${ }^{2}$, YOULIANG GAO ${ }^{1}$, BINGYING XIE ${ }^{1}$, GUANGHONG HU ${ }^{1}$, WENYI FANG ${ }^{1}$, \\ JUN PENG ${ }^{3}$, YOUQIN CHEN ${ }^{4}$ and THOMAS J. SFERRA ${ }^{4}$ \\ ${ }^{1}$ Department of Gastroenterology, Second Affiliated Hospital of Fujian University of Traditional Chinese Medicine; \\ ${ }^{2}$ Fujian Institute of Chinese Medicine, Fuzhou, Fujian 350003; ${ }^{3}$ Academy of Integrative Medicine, \\ Fujian University of Traditional Chinese Medicine, Fuzhou, Fujian 350122, P.R. China; ${ }^{4}$ Rainbow Babies \\ and Children's Hospital, Case Western Reserve University School of Medicine, Cleveland, OH 44106, USA
}

Received May 14, 2013; Accepted July 24, 2013

DOI: $10.3892 /$ ijmm.2013.1458

\begin{abstract}
The activation of the Toll-like receptor 4

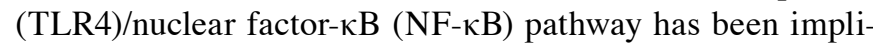
cated as a key mediator in the pathogenesis of ulcerative colitis (UC); therefore, it has become an attractive target for the treatment of UC. Qing Hua Chang Yin (QHCY) is a traditional Chinese formula, which has been used for many years to clinically treat conditions associated with inflammatory bowel diseases, such as UC. However, the precise mechanisms behind its anti-inflammatory effects remain largely unknown. In this study, using the dextran sulfate sodium (DSS)-induced colitis mouse model, we evaluated the therapeutic effects of QHCY against UC and elucidated the possible underlying molecular mechanisms. We found that the administration of QHCY profoundly ameliorated DSS-induced clinical manifestations, colon shortening and histological damage in the mice with colitis. In addition, treatment with QHCY significantly decreased the DSS-induced secretion of serum amylase. Moreover, QHCY significantly inhibited the DSS-induced expression of TLR4 and myeloid differentiation primary response gene 88 (MyD88), the phosphorylation of $\mathrm{I} \kappa \mathrm{B}$ and the nuclear translocation of NF- $\mathrm{kB}$. Taken together, our findings suggest that the suppression of the TLR4/NF- $\kappa \mathrm{B}$ signaling pathway may be one of the mechanisms involved in the therapeutic effects of QHCY against UC.
\end{abstract}

Correspondence to: Dr Xiao Ke, Department of Gastroenterology, Second Affiliated Hospital of Fujian University of Traditional Chinese Medicine, 13 Hu Dong Zhi Road, Fuzhou, Fujian 350003, P.R. China

E-mail: drkxkx@163.com

Abbreviations: QHCY, Qing Hua Chang Yin; TCM, traditional Chinese medicine; UC, ulcerative colitis; IBD, inflammatory bowel disease; NF- $\kappa \mathrm{B}$, nuclear factor- $\kappa \mathrm{B}$; TLR4, Toll-like receptor 4

Key words: Qing Hua Chang Yin, traditional Chinese medicine, ulcerative colitis, Toll-like receptor $4 /$ nuclear factor- $\kappa \mathrm{B}$ pathway

\section{Introduction}

Inflammatory bowel disease (IBD), which is a term used to describe a group of inflammatory conditions, such as ulcerative colitis (UC) and Crohn's disease (CD), is associated with chronically relapsing disorders of the gastrointestinal tract (1-3). Histologically, it is characterized by the presence in the gut of extensive areas of ulceration, pronounced infiltration of neutrophils and epithelial cell necrosis. Although these conditions have been treated with 5-aminosalicylic acid derivatives, corticosteroids and immunosuppressants, such as azathioprine and cyclosporine (1), few non-toxic therapeutic options are currently able to modulate intestinal inflammation. Therefore, the challenge remains to develop novel and specific therapies for IBD (4).

Nuclear factor- $\kappa \mathrm{B}(\mathrm{NF}-\kappa \mathrm{B})$ activation is one of the most important events involved in the pathogenesis of $\operatorname{IBD}(5,6)$. NF- $\kappa$ B can be activated by several upstream stimuli, including Toll-like receptors (TLRs). The transmembrane TLRs are a family of pattern-recognition receptors (PRRs) that enable the innate and adaptive immune systems to recognize pathogen-associated molecular patterns (PAMPs). Thus far, over 13 members of the TLR family have been identified in mammals, of which TLR4 is the most extensively studied (7). TLR4 can be activated by recognizing various PAMPs in bacteria $(8,9)$. Upon ligand binding, TLR4 undergoes a conformational change and dimerizes, recruiting adaptor proteins, such as myeloid differentiation primary response gene 88 (MyD88), which in turn activates NF- $\mathrm{kB}$, transducing the immune-related signals to the nucleus $(10,11)$. Due to its essential role in the pathogenesis of inflammatory diseases, including IBD (12-18), modulation of the TLR4/NF- $\kappa B$ signaling pathway may be a main target for the treatment of inflammation.

Natural products, such as those found in traditional Chinese medicine (TCM), have received much attention due to their anti-inflammatory potential. Qing Hua Chang Yin (QHCY) is a well-known traditional Chinese formula consisting of a combination of 11 herbs, including Herba et Gemma Agrimoniae, Coptis chinensis Franch, Radix Sanguisorbae, Radix Paeoniae Rubra, Elettaria cardamomum, Magnolia officinalis, Artemisia 
capillaris Thunb., Herba Eupaatorii Fortunei, Semen Coicis, Semen Dolichoris Album and Poria cocos. In conjuction, these components confer QHCY properties, such as eliminating heat and dampness, and strengthening the spleen, thus increasing vitality (tonifying the Spleen Qi in Chinese). According to TCM theory, the accumulation of toxic dampness and heat is one of the major causative factors in the pathogenesis of UC. QHCY therefore has long been used in China to clinically treat UC (19-24). However, the precise mechanisms behind the therapeutic effects of QHCY against UC remain largely unknown. Thus, in the present study we evaluated the therapeutic effects of QHCY on an established mouse model of colitis induced by dextran sulfate sodium (DSS) and investigated the possible molecular mechanisms involved.

\section{Materials and methods}

Materials and reagents. DSS (average molecular weight, 36,000-50,000) was purchased from MP Biochemicals (Solon, $\mathrm{OH}, \mathrm{USA}$ ). The BCA Protein assay and nuclear protein extraction kits were purchased from Beyotime Institute of Biotechnology (Shanghai, China). Antibodies for western blot analysis were obtained from Cell Signaling Technology (Beverly, MA, USA). The mouse serum amyloid A (SAA) ELISA kit was obtained from Immunology Consultants Laboratory, Inc. (Newberg, OR, USA). All the other chemicals, unless otherwise stated, were obtained from Sigma-Aldrich (St. Louis, MO, USA).

Preparation of QHCY. In total, $220 \mathrm{~g}$ dehydrated Herba et Gemma Agrimoniae, $33 \mathrm{~g}$ dehydrated Coptis chinensis Franch, 100 g dehydrated Radix Sanguisorbae, 110 g dehydrated Radix Paeoniae Rubra, 56 g dehydrated Elettaria cardamomum, $110 \mathrm{~g}$ dehydrated Magnolia officinalis, $110 \mathrm{~g}$ dehydrated Artemisia capillaris Thunb, 110 g dehydrated Herba Eupaatorii Fortunei, 220 g dehydrated Semen Coicis, $110 \mathrm{~g}$ dehydrated Semen Dolichoris Album and $220 \mathrm{~g}$ dehydrated Poria cocos were extracted with boiling water 3 times. The extracts were then combined and concentrated by boiling to a final volume of $1,000 \mathrm{ml}$. The final concentration of QHCY crude drug was $\sim 1.4 \mathrm{mg} / \mathrm{ml}$.

Establishment of mouse model of colitis and QHCY treatment. Male BALB/c mice (with an initial body weight of 20-22 g) were obtained from Shanghai SLAC Laboratory Animal Co., Ltd. (Shanghai, China) and housed under pathogen-free conditions with a $12 \mathrm{~h}$ light/dark cycle. Food and water were provided ad libitum and the mice were allowed to acclimatize for 1 week prior to the experiment. Housing conditions and all animal experiments were approved by the Institutional Animal Care and Use Committee of Fujian University of Traditional Chinese Medicine, Fuzhou, China. Colitis was induced by the administration of $3 \%$ DSS (weight to volume ratio dissolved in distilled water) in the drinking water for 8 days. On the first day of model construction, the animals were randomly divided into 3 groups $(n=10)$ : the normal control group in which the mice received neither DSS stimulation nor QHCY treatment; and the DSS-induced UC model or QHCY-treated group in which the mice received DSS stimulation and then received an intragastric administration of $200 \mu \mathrm{l}$ of saline or QHCY, respectively, daily for 12 days.
Table I. Disease activity index score.

\begin{tabular}{lccl}
\hline Score & Weight loss $(\%)$ & Stool consistency & Rectal bleeding \\
\hline 0 & 0 & Normal & Normal \\
1 & $1-5$ & & \\
2 & $6-10$ & Loose & Hemoccult-positive \\
3 & $11-20$ & & \\
4 & $>21$ & Diarrhea & Gross bleeding \\
\hline
\end{tabular}

The disease activity index was calculated as the sum of scores for weight loss, stool consistency and rectal bleeding.

Evaluation of clinical manifestations. The progression of DSS-induced colitis was monitored daily in a blinded manner, including the observation of changes in body weight, stool consistency and the presence of rectal bleeding blood in the stool. The disease activity index (DAI) score was calculated as the sum of scores for weight loss, stool consistency and rectal bleeding as previously described (25) (Table I).

Sample collection. At the end of the experiments, the animals were anesthetized and blood was collected via right heart ventricle puncture in lightly heparinized syringes and kept on ice. Sera were separated by 5 min centrifugation at $5,000 \times \mathrm{g}$ and stored at $-80^{\circ} \mathrm{C}$ prior to the analysis. The colons were excised and the length from the cecum to the anus was measured. One portion of each distal colon was cut and fixed in $10 \%$ formalin for histological examination; the remainder was used for further analysis.

Histopathological examination. Small pieces of the colon tissues were fixed with $10 \%$ buffered formalin for $24 \mathrm{~h}$. Samples were then paraffin-embedded, sectioned and stained with hematoxylin and eosin (H\&E). Histopathological changes were observed under a light microscope.

Measurement of SAA levels by ELISA. The level of SAA in the sera was measured using a mouse SAA ELISA kit according to the manufacturer's instructions. All samples were assayed in triplicate. The concentrations of SAA were determined by comparison to serial dilutions of SAA purified standard.

Western blot analysis. Five fresh colon tissues were selected randomly from each group, homogenized in non-denaturing lysis buffer using homogenizer and centrifuged at 15,000 x g for $15 \mathrm{~min}$. Nuclear proteins were extracted using the Nuclear Protein Extraction kit (Beyotime Institute of Biotechnology) according to the manufacturer's instructions. Protein concentrations were determined using the BCA protein assay kit. Equal amounts of protein from each sample were resolved on $12 \%$ Tris-glycine gels and transferred onto PVDF membranes. The membranes were blocked for $2 \mathrm{~h}$ with $5 \%$ non-fat dry milk and incubated with the desired primary antibodies

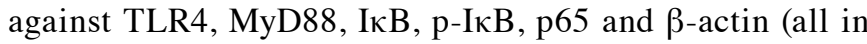
1:1,000 dilutions) overnight at $4^{\circ} \mathrm{C}$ and then with appropriate HRP-conjugated secondary antibody followed by enhanced chemiluminescence detection. 


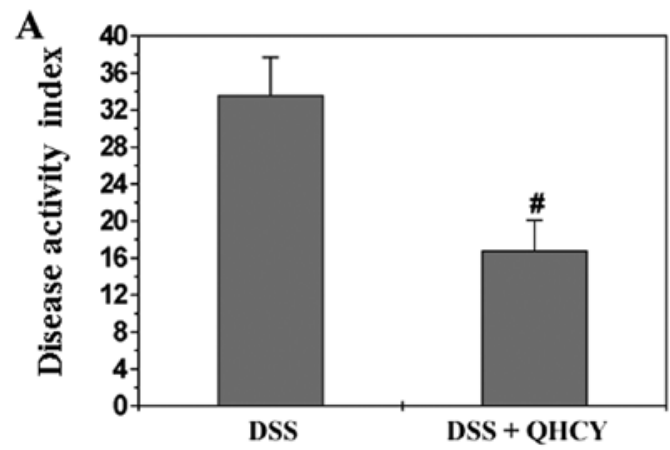

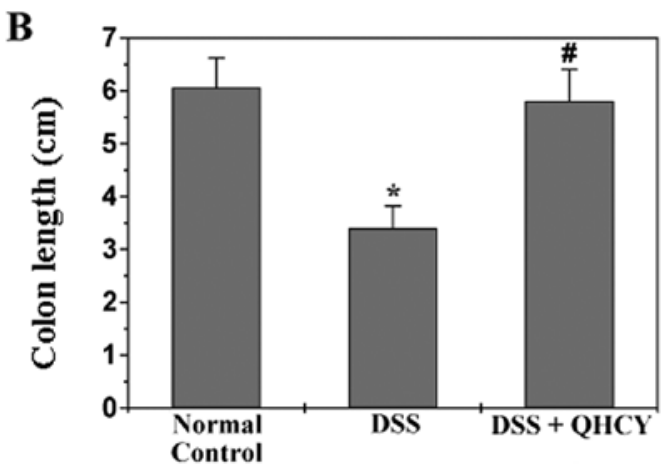

Figure 1. Effect of Qing Hua Chang Yin (QHCY) on the clinical manifestations in the dextran sulfate sodium (DSS)-induced ulcerative colitis mouse model. Colitis was induced by the administration of DSS. On the first day of model construction, the animals received an intragastric administration of saline or QHCY. daily for 12 days. (A) The progression of DSS-induced colitis was monitored daily in a blinded manner, including the observation of changes in body weight, stool consistency and rectal bleeding. The disease activity index (DAI) was calculated as described in Table I. (B) At the end of the experiments, the animals were anesthetized. The colons were excised and the length from the cecum to the anus was measured. Data shown are the means \pm SD (error bars) from 10 individual mice/group. ${ }^{*} \mathrm{P}<0.05$, vs. normal controls; ${ }^{*} \mathrm{P}<0.05$, vs. mice stimulated with DSS but not treated with QHCY.
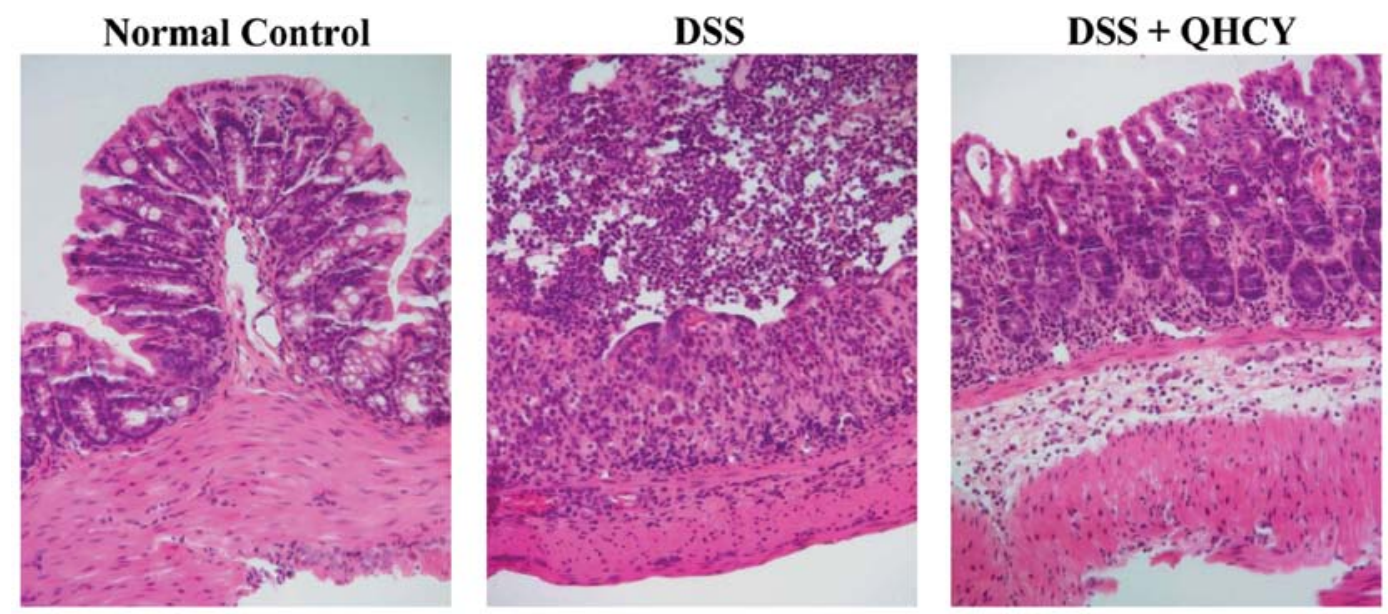

Figure 2. Effect of Qing Hua Chang Yin (QHCY) on histological damages in colon tissues in the dextran sulfate sodium (DSS)-induced ulcerative colitis mouse model. Colon tissues were stained with hematoxylin and eosin. Histopathological changes were observed under a microscope. Normal control mice displayed normal colonic histology with an intact epithelium, well-defined gland lengths and no leukocyte infiltration in the mucosa. DSS stimulation resulted in mucosal ulceration, infiltration of inflammatory cells, crypt distortion and a hyperplastic epithelium. However, the DSS-induced histological damages in the colon tissues were significantly ameliorated following treatment with QHCY. Representative images are shown (magnification, x100).

Statistical analysis. Data were analyzed using the SPSS package for Windows (v11.5). Statistical analysis of the data was performed using the Student's t-test and one-way ANOVA. P-values $<0.05$ were considered to indicate statistically significant differences.

\section{Results}

QHCY improves the clinical manifestations in the DSS-induced UC mouse model. To evaluate the therapeutic efficacy of QHCY against the development of mouse colitis, clinical manifestations, such as changes in weight, stool consistency and rectal bleeding were observed and the DAI was calculated. As shown in Fig. 1, compared with the normal group, mice in the DSS-stimulated group displayed obvious manifestations of UC, including body weight loss, diarrhea and rectal bleeding, indicating the successful construction of the model. However, treatment with QHCY significantly improved the DSS-induced manifestations. The DAI score of the normal control, DSS-stimulated model or QHCY-treated group was $0,11.2 \pm 1.79$ and $5.6 \pm 1.14$, respectively $(\mathrm{P}<0.05)$ (Fig. 1A). To verify these results, the colons of mice from each group were harvested after sacrifice and the length from the cecum to the anus was measured. As shown in Fig. 1B, DSS stimulation resulted in significant colon shortening, which however was profoundly neutralized following treatment with QHCY. The average colon length per mouse from the normal control, model and QHCY-treated group was 6.06 $\pm 0.56,3.4 \pm 0.42$ and $5.8 \pm 0.60 \mathrm{~cm}$, respectively $(\mathrm{P}<0.05)$. Taken together, these data demonstrate the therapeutic efficacy of QHCY against UC.

QHCY ameliorates histological damage of colon tissue in the DSS-induced UC mouse model. The histological changes of the colonic mucosa in the experimental mice were observed under a light microscope after H\&E staining. As shown in Fig. 2, the normal control mice displayed normal colonic histology with an intact epithelium, well-defined gland lengths and no leukocyte infiltration in the mucosa. DSS stimulation resulted 


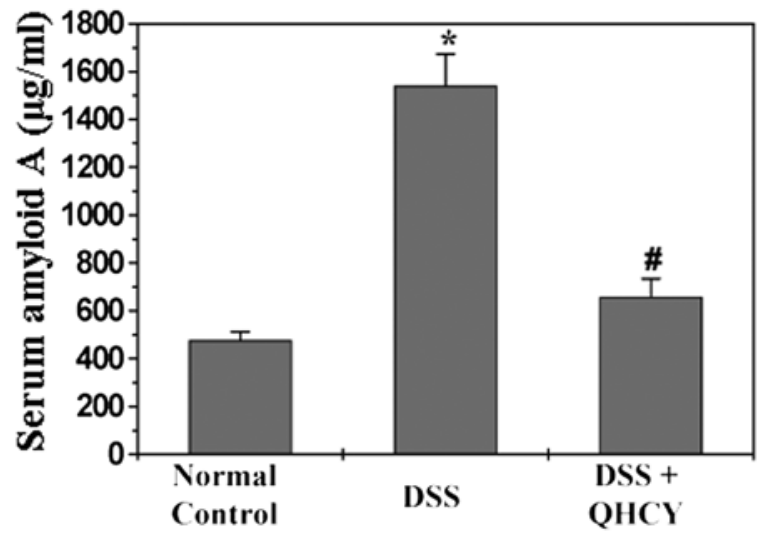

Figure 3. Effect of Qing Hua Chang Yin (QHCY) on the level of serum amyloid A (SAA) in the dextran sulfate sodium (DSS)-induced ulcerative colitis mouse model. The levels of SAA in sera were determined by ELISA. Data shown are the means \pm SD (error bars) from 10 individual mice/group. ${ }^{*} \mathrm{P}<0.05$, vs. normal controls; ${ }^{\text {}} \mathrm{P}<0.05$, vs. mice stimulated with DSS but not treated with QHCY.

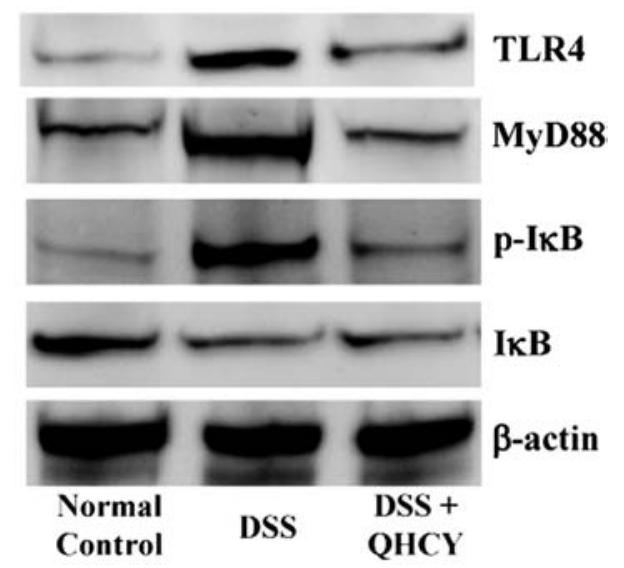

Figure 4. Effect of Qing Hua Chang Yin (QHCY) on the expression of Toll-like receptor 4 (TLR4), myeloid differentiation primary response gene 88 (MyD88) and $\mathrm{I} \kappa \mathrm{B}$ phosphoralytion in the dextran sulfate sodium (DSS)-induced ulcerative colitis mouse model. TLR4 and MyD88 expression, as well as $\mathrm{I} \kappa \mathrm{B}$ phosphorylation in colon tissues were determined by western blot analysis. $\beta$-actin was used as the internal control. Blots are representative of colon tissues randomly selected from 5 individual mice in each group.

in mucosal ulceration, infiltration of inflammatory cells, crypt distortion and a hyperplastic epithelium. However, the DSS-induced histological damages in the colon tissues were significantly ameliorated following treatment with QHCY.

QHCY reduces the levels of SAA in the DSS-induced UC mouse model. SAA, one of the inflammatory markers, has been shown to be elevated in patients with UC. We therefore examined the serum levels of SAA in the experimental mice by ELISA. As shown in Fig. 3, the serum levels of SAA in the UC model mice were significantly higher than those of the mice in the normal control group $(\mathrm{P}<0.05)$. The administration of QHCY significantly inhibited the DSS-induced increase in the serum levels of SAA $(\mathrm{P}<0.05)$.

QHCY suppresses the activation of the TLR4/NF- $\kappa B$ signaling pathway in the DSS-induced UC mouse model. To elucidate

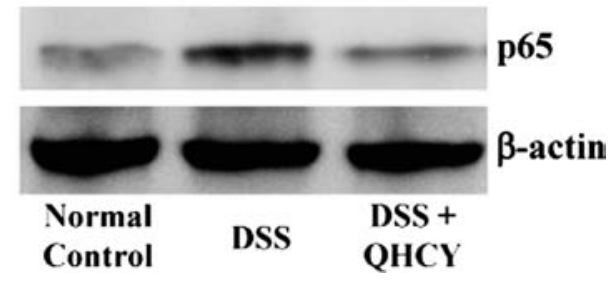

Figure 5. Effect of Qing Hua Chang Yin (QHCY) on nuclear factor- $\kappa \mathrm{B}$ $(\mathrm{NF}-\kappa \mathrm{B})$ nuclear translocation in the dextran sulfate sodium (DSS)-induced ulcerative colitis mouse model. The nuclear content of the NF- $\mathrm{BB}$ p65 subunit in colon tissues was determined by western blot analysis. $\beta$-actin was used as the internal control. Blots are representative of colon tissues randomly selected from 5 individual mice in each group.

the mechanisms behind the therapeutic effects of QHCY against UC, we determined its effects on the activation of the TLR4/NF- $\kappa$ B pathway in colon tissues of mice with UC. As shown in Fig. 4, the expression of TLR4 and MyD88, as well as the phosphorylation level of $\mathrm{I} \kappa \mathrm{B}$ were significantly increased in the DSS-induced UC model group compared with those in the normal control group; however, these levels were neutralized following treatment with QHCY. We also examined alterations in the nuclear content of the NF- $\mathrm{NB}$ p65 subunit by western blot analysis, in order to evaluate the effects of QHCY on NF- $\kappa \mathrm{B}$ nuclear translocation, which is a critical step for $\mathrm{NF}-\kappa \mathrm{B}$ activation. As shown in Fig. 5, QHCY significantly inhibited the DSS-induced nuclear translocation of p65 in the colon tissues of mice with UC.

\section{Discussion}

The use of natural anti-inflammatory products provides an attractive and relatively non-toxic alternative remedy to control inflammatory disorders. As a well-known traditional Chinese formula, QHCY has long been used in China to clinically treat UC, a major form of IBD (19-24). However, the precise mechanisms behind the inhibitory effects of QHCY on intestinal inflammation remain largely unclear.

In the present study, we evaluated the therapeutic effects of QHCY against UC using an experimental mouse model of DSS-induced colitis. We found that treatment with QHCY significantly improved the DSS-induced clinical manifestations, as evidenced by the prevention of body weight loss, as well as the alleviation of diarrhea and rectal bleeding in mice with UC. In addition, treatment with QHCY profoundly neutralized colon shortening and ameliorated colonic histological damages in the DSS-induced colitis mouse model. Moreover, QHCY significantly inhibited the DSS-induced increase in serum levels of SAA, one of the inflammatory markers which is commonly overexpressed in patients with UC. Taken together, these data demonstrate the therapeutic efficacy of QHCY against the development of UC.

The TLR4/NF- $\kappa \mathrm{B}$ signaling pathway is one of the major pathways mediating inflammatory responses. TLR4 is activated by recognizing PAMPs in bacteria, which in turn triggers signaling cascades leading to the activation of NF- $\kappa \mathrm{B}$ (12). TLR4 is expressed at low levels in normal intestines but is increased in mice with DSS-induced colitis $(13,14)$ and in patients with $\operatorname{IBD}(15,16)$, which may contribute to the 
initiation or perpetuation of intestinal inflammation. Previous studies have demonstrated that TLR4 functions as a mediator of intestinal inflammation (17) and that the blockade of TLR4 ameliorates DSS-induced colitis (18). Thus, the TLR4/NF- $\mathrm{B}$ pathway has become a major target for the treatment of inflammatory diseases, including UC. The processes of TLR4/NF- $\mathrm{BB}$ activation include several key links, such as MyD88 attendance, phosphorylation/degradation of I $\mathrm{B}$ and the subsequent nuclear translocation of NF- $\mathrm{NB}$. In this study, to elucidate the mechanisms behind the therapeutic effects of QHCY against UC, we examined its effects on the TLR4/NF- $\kappa \mathrm{B}$ signaling pathway. We found that treatment with QHCY significantly inhibited the expression of TLR4 and MyD88, as well as the phosphorylation of $\mathrm{I} \kappa \mathrm{B}$ and blocked NF- $\kappa \mathrm{B}$ nuclear translocation in the colon tissues of mice with UC, suggesting that QHCY suppresses the activation of the NF- $\mathrm{KB}$ signaling pathway.

In conclusion, to our knowledge, in this study, we demonstrate for the first time that QHCY prevents the development of UC in vivo through the suppression of the TLR4/NF- $\mathrm{B}$ signaling pathway. These results provide further fundamental evidence for QHCY as a therapeutic agent for the treatment of UC.

\section{Acknowledgements}

The present study was sponsored by a grant from the National Natural Science Foundation of China (81173432).

\section{References}

1. Rezaie A, Parker RD and Abdollahi M: Oxidative stress and pathogenesis of inflammatory bowel disease: an epiphenomenon or the cause? Dig Dis Sci 52: 2015-2021, 2007.

2. Podolsky DK: Inflammatory bowel disease. N Engl J Med 347: 417-429, 2002.

3. Odashima M, Otaka M, Jin M, et al: Successful treatment of refractory duodenal Crohn's disease with infliximab. Dig Dis Sci 52: 31-32, 2007.

4. Isaacs KL, Lewis JD, Sandborn WJ, et al: State of the art: IBD therapy and clinical trials in IBD. Inflamm Bowel Dis 11 (Suppl 1): S3-S12, 2005.

5. Schreiber S, Nikolaus S and Hampe J: Activation of nuclear factor kappa B inflammatory bowel disease. Gut 42: 477-484, 1998.

6. Andresen L, Jørgensen VL, Perner A, Hansen A, Eugen-Olsen J and Rask-Madsen J: Activation of nuclear factor kappaB in colonic mucosa from patients with collagenous and ulcerative colitis. Gut 54: 503-509, 2005.

7. Kawai T and Akira S: TLR signaling. Cell Death Differ 13: $816-825,2006$
8. Boone DL and Ma A: Connecting the dots from Toll-like receptors to innate immune cells and inflammatory bowel disease. J Clin Invest 111: 1284-1286, 2003.

9. Janeway CA Jr and Medzhitov R: Innate immune recognition. Annu Rev Immunol 20: 197-216, 2002.

10. Bowie A and O'Neill LA: The interleukin-1 receptor/Toll-like receptor superfamily: signal generators for pro-inflammatory interleukins and microbial products. J Leukoc Biol 67: 508-514, 2000.

11. Slack JL, Schooley K, Bonnert TP, et al: Identification of two major sites in the type I interleukin-1 receptor cytoplasmic region responsible for coupling to pro-inflammatory signaling pathways. J Biol Chem 275: 4670-4678, 2000.

12. Pålsson-McDermott EM and O'Neill LA: Signal transduction by the lipopolysaccharide receptor, Toll-like receptor- 4 . Immunology 113: 153-162, 2004.

13. Ohkawara T, Takeda H, Miyashita K, et al: Regulation of Toll-like receptor 4 expression in mouse colon by macrophage migration inhibitory factor. Histochem Cell Biol 125: 575-582, 2006.

14. Ortega-Cava CF, Ishihara S, Rumi MA, et al: Strategic compartmentalization of Toll-like receptor 4 in the mouse gut. J Immunol 170: 3977-3985, 2003.

15. Hausmann M, Kiessling S, Mestermann S, et al: Toll-like receptors 2 and 4 are up-regulated during intestinal inflammation. Gastroenterology 122: 1987-2000, 2002.

16. Szebeni B, Veres G, Dezsõfi A, et al: Increased expression of Toll-like receptor (TLR) 2 and TLR4 in the colonic mucosa of children with inflammatory bowel disease. Clin Exp Immunol 151: 34-41, 2008

17. Fukata M, Chen A, Klepper A, et al: Cox-2 is regulated by Toll-like receptor- 4 (TLR4) signaling: role in proliferation and apoptosis in the intestine. Gastroenterology 131: 862-877, 2006.

18. Ungaro R, Fukata M, Hsu D, et al: A novel Toll-like receptor 4 antagonist antibody ameliorates inflammation but impairs mucosal healing in murine colitis. Am J Physiol Gastrointest Liver Physiol 296: G1167-G1179, 2009.

19. Wang XY and Tian DL: Etiological and pathological characteristics of ulcerative colitis and TCM differentiation and treatment. Beijing Zhong Yi Yao Da Xue Xue Bao 30: 554-559, 2007 (In Chinese).

20. Gong YP, Liu W, Ma GT, et al: Randomized control study of 'Qingchang Suppository' on ulcerative colitis. Shanghai Zhong Yi Yao Da Xue Xue Bao 21: 33-36, 2007 (In Chinese).

21. Fu NL and Huang JY: Progress of clinical research of traditional Chinese medicine for the treatment of ulcerative colitis. Journal of Traditional Chinese Medicine 40: 501-503, 1999 (In Chinese).

22. Li QG: An idea about treatment of ulcerative colitis by TCM methods. Beijing Zhong Yi 23: 149-150, 2004 (In Chinese).

23. Wang CH, Gao WY, Li YF, et al: Study of Fufangkushen colon-release capsule on ulcerative colitis of endo-retention of damp heat type. Xian Dai Zhong Xi Yi Jie He Za Zhi 18: 13-15, 2009 (In Chinese).

24. Chen JT, Ke X, Fu XY, et al: The clinical study of heat-clearing and damp-drying on the treatment of damp-heat ulcerative colitis. Zhongguo Zhong Xi Yi Jie He Xiao Hua Za Zhi 17: 256-257, 2009 (In Chinese).

25. Cooper HS, Murthy SN, Shah RS and Sedergran DJ: Clinicopathologic study of dextran sulfate sodium experimental murine colitis. Lab Invest 69: 238-249, 1993. 